\title{
Modelling of Endpoint Feedback Learning Implemented Through Point-to-Point Learning Control
}

\author{
Shou-Han Zhou, Member, IEEE, Ying Tan, Senior Member, IEEE, Denny Oetomo, Senior Member, IEEE, \\ Chris Freeman, Member, IEEE, Etienne Burdet, Member, IEEE, and Iven Mareels, Fellow, IEEE
}

\begin{abstract}
In the last decade, several experiments were conducted to investigate human motor control behaviour for the task of arm reaching, using the visual feedback of their hand position provided only at the end of each trial (of reaching motion). Current computational frameworks so far have yet to model the observations made in these experiments that humans learn to complete a task by feedforward action using such feedback, which is considered as a displacement error at the end of each trial of the reaching motion. This paper demonstrates how this learning can be formulated as an optimization problem, and hence can be modelled by an optimal control based design framework. By designing a cost function which weighs the tracking of the target and the smoothness of human motion, the constructed framework, implemented in the form of point-to-point learning control (LC), inherently embeds the feedforward control and enables learning over the repeated trials using the errors only at the endpoint of a reaching motion trajectory, as opposed to that of the entire reference trajectory; and is therefore able to reproduce the human learning behaviour observed in the experiments.
\end{abstract}

Index Terms-Iterative Learning Control, Computational Neuroscience, Human Motor Learning

\section{INTRODUCTION}

Humans are observed to successfully accomplish their given tasks when provided with the continuous responses from muscle dynamics as well as the visual and proprioceptive feedback of their movement [5], [13], [20], [29], [33]. To study the motor control involved in accomplishing these tasks, experiments have been developed in which humans are required to perform a task successfully in a controlled environment. Accompanying these experiments are computational models which aim at explaining the experimental results and the underlying mechanism [8], [28], [34].

A particular class of experiments of recent interest are those which attempt to assess human motor learning dynamics in reaching tasks where continuous visual feedback is not provided during the motion, forcing the human subjects to learn using only visual feedback provided at the end of each iterative movement trial [6], [9], [14], [23]. The results of these experiments show that learning in a target reaching task is not affected whether the subjects are presented with visual feedback across the entire trajectory; as long as the endpoint

Y. Tan, D. Oetomo and I. Mareels are with Melbourne School of Engineering, the University of Melbourne, Parkville, VIC 3010, Australia. yingt, doetomo, i.mareelsaunimelb.edu.au,. C. Freeman is with the School of Electronics and pon puter Science, University of Southampton, Southampton, SO17 1BJ, UK ?. H. Zhou and E. Burdet are with Department of Bioengineering, Imperial College London, UK. This work is supported by the Australian Research Council (ARC) under Future Fellow Project: FT0991385, the Discovery Project: DP130100849 and the Diseovery Project: DP160104018; and is supperted by the EU Horizon 2020 project, CogIMon.

Manuscript received June 28, 2016; accepted on September 03, 2016. position is provided after the movement is completed [9]. It is hypothesized from these results that when continuous feedback is not provided, humans use a feedforward strategy to perform movements [11], [12], [30], while employing an error-based learning method to improve task performance across trials [32].

Despite the many experiments having been carried out in this manner, a computational modelling for the process of motor learning using endpoint information has yet to be constructed. Current models of human motor control with endpoint vision use a forward prediction model to simulate the movements [1], [15], [19], [31]. However, such models do not explain how humans are able to learn a feedforward control action using the visual error information at the end of the trials. On the other hand, models which focus on learning are used to capture the human learning behaviour observed in experiments in which continuous feedback during the arm reaching movement is available [4], [8], [34]. Therefore, these models require a reference trajectory that is defined at every sampling instance throughout the duration of the motion, and are not representative of experiments in which continuous feedback is not available.

Considering the above, this paper aims to present a novel framework to model human learning when visual feedback is provided only at the end of each reaching movement; based on the notion that humans learn a feedforward control action using the visual error information provided only at the end of their movement.

In this paper, it is demonstrated that the human learning behaviour using endpoint visual feedback can be formulated as an optimization problem. By solving this problem using suitable optimization-based techniques, a framework can be subsequently constructed to model the human motor learning. Although there exist many optimization-based control frameworks in the literature, such as [21], [27] to name a few, none has been applied to simulate human learning using endpoint visual feedback. Considering this, the current paper demonstrates how a framework for modelling human learning using endpoint vision can be constructed by solving the aforementioned optimization problem using point-to-point learning control (LC) [10], which has previously been used to capture the behaviour of a human individual learning the task of reaching using continuous visual feedback during the whole movement [35]. By doing so, the framework is shown to be able to capture the observation that human learning occurs in the cases where feedback is provided throughout the entire movement as well as where feedback is provided only at the endpoint [9]. 
This paper is organized as follows. First, a simpler variation of the experiments reported in [33] is presented in Section II. The conducted experiment shows that humans can learn the task of reaching when provided with only endpoint visual feedback. The experimental results are subsequently used to formulate the modelling of human learning using the endpoint feedback as a particular optimization problem in Section III. Section IV then proceeds to solve the proposed optimization problem, where point-to-point LC is used as a specific method of solving the problem. Two sets of simulation results (with and without the consideration of the smoothness of human reaching motion) are co red to the experiment results in Section V. It is worthw to note that the purpose of the simulations is not to produce a perfect model of human motion but rather to illustrate the ability of the framework in modelling human learning using endpoint information. The features of the framework, its limitations and future applications are discussed in Section VI.

\section{Human LEARNING USING ENDPOINT FEEDBACK}

The capability of humans to complete a task using feedforward action learnt only through feedback provided at the endpoint of each iteration of a repetitive exercise has been previously demonstrated by experiments reported in [26]. The experiment described in this section is a simpler variation of that reported in [26], exhibiting the human ability to learn to reach a given target point when presented with only the final cursor position at the end of each trial, and thereby motivates the need for a computational framework of human motor learning for such cases. The experimental results presented serve as a means of validating the performance of the model in capturing human learning under the experimental conditions.

The equipment used in the setup is first described, followed by the task to be performed by the subjects and then the experimental conditions. Interested readers should refer to [33] for further details.

\section{A. Equipment Setup}

Experiment Equipment: The experiment setup is comprised of a the robot (3DOM) [18] (Figure 1).

During the experiment, the human subject holds onto the robot arm while seated on a high chair, facing a mirror positioned at shoulder level which serves to prevent direct visual feedback of the hand and of the robotic arm. The movement of the subject's hand position during the experiment is tracked by the robot end-effector at a sampling rate of $200 \mathrm{~Hz}$. The position is obtained by the software to determine the location of a $1 \mathrm{~cm}$ diameter cursor on an LCD monitor resting on a platform above the subject, which is reflected in the mirror.

General Experiment Description: Six neurologically unimpaired, naïve subjects, (four right-handed males, one female, one left-handed male), were invited to participate in the experiment. During the experiment, the subjects performed point-to-point planar reaching movements to a target point. More specifically, the subjects were required to reach from a starting point towards a target ring $20 \mathrm{~cm}$ away from the
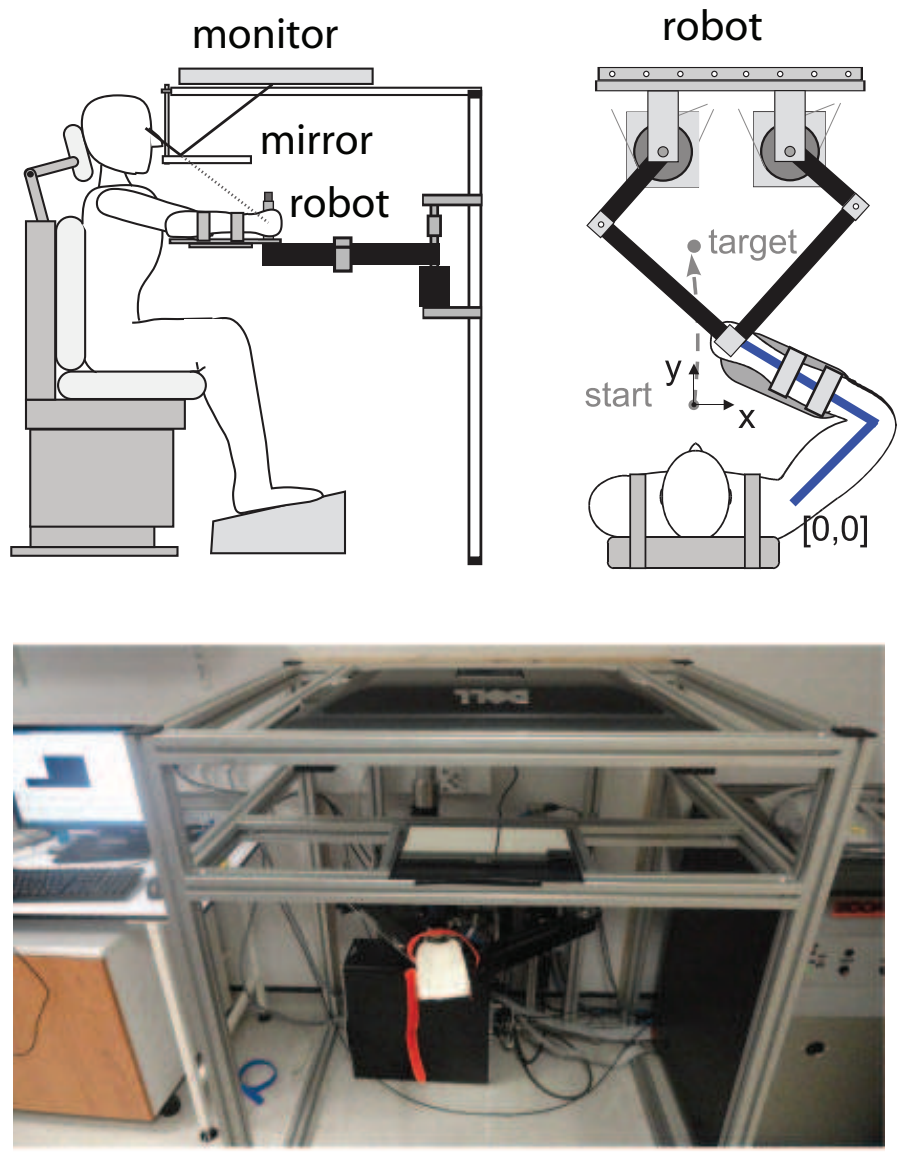

Fig. 1. Experimental setup showing the subject holding onto the robot. The subject's hand is constrained to the robot by a cuff. For the task of pointto-point reaching, the target and the cursor representing the subject's hand is displayed on a monitor. The subject observes a reflection of the screen on a mirror which also serves to separate the subject's hand from his/her vision.

body parallel to the sagittal plane along the $y$ axis within $550 \pm 100 \mathrm{~ms}$ (Figure 1). The timer started when the subjects' velocity exceeded $0.4 \mathrm{~m} / \mathrm{s}$ and stopped when the velocity was less than $0.4 \mathrm{~m} / \mathrm{s}$ for more than $10 \mathrm{~ms}$.

At the end of each movement, the subjects were shown the final cursor location and the target ring would change to one of red, green or blue, depending on whether the subjects were too slow, too fast or on time in arriving at the target, respectively. After about 1.2 seconds, the cursor disappeared and the robot assisted the subject back to the starting point for the next trial. A scoring system which rewards the subject for arriving at the target on time was applied to the experiment.

Experiment Protocol: Before the experiment, the subjects were provided with practice trials of 100 movements reaching for the target while being provided with continuous feedback of the cursor position throughout each trial. The time to finish the reaching tasks was not restricted.

During the experiment, the subjects were required to perform the same reaching movement with continuous visual feedback removed. The subjects were aware of the visual feedback being removed from their vision. They were also aware that the requirement for the task was now to perform 


\section{Experiment Results}
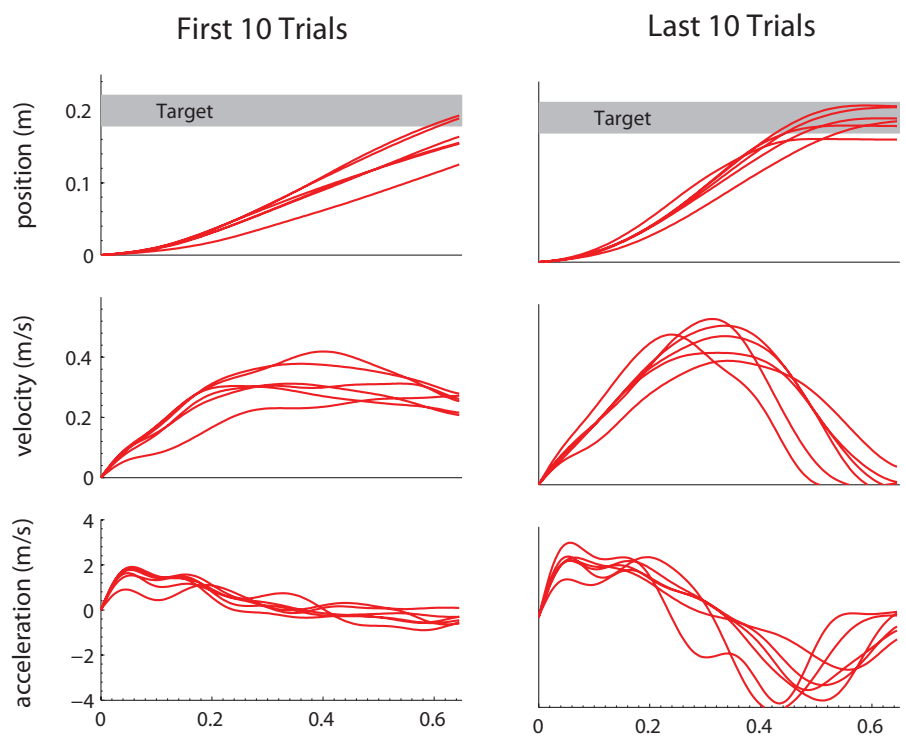

time (s)

Fig. 2. A typical subject's position, velocity and acceleration profiles during the experiment. Left column shows the first 10 trials during which the visual cursor is turned off. The right column shows the last 10 trials the subject makes before the experiment is finished. This ensures that the subject reached a steady-state in achieving the point-to-point reaching task given visual information at the end of each trial as required by the experiment.

the movement in $550 \pm 100 \mathrm{~ms}$. The subjects were required to perform 30 successful trials in order to complete the experiment. The subjects' learning behaviour in these trials were recorded and are presented in the following section.

\section{B. Experiment Results}

All six subjects were able to perform the task successfully. A typical subject's trajectory profile (Subject 1 ) along the $y$ direction for this reaching task is shown in Figure 2. From the results, it is observed that for the first 10 trials, the subjects performed smooth movements with low velocity and were not able to arrive at the target with zero velocity in $550 \mathrm{~ms}$ (Figure 2, left column). For the last 10 trials of the experiment, the subjects showed that they were able to arrive at the target with zero velocity in $550 \mathrm{~ms}$ (Figure 2, column). Furthermore, the movements exhibited a bell-sha profile, in agreement with those found in the literature [13]. Therefore, it was observed that the subjects learned to improve their performance during the experiment using the endpoint feedback provided to them at the end of each trial [26].

By plotting the subject's position at $550 \mathrm{~ms}$, it is seen in Figure $3 \mathrm{~A}$ that the subject learns to improve his/her performance such that his/her position at $550 \mathrm{~ms}$ converges to the target position.

In addition, the obtained experimental data was fitted to an exponential function

$$
y(k)=a \exp (b k),
$$

A.
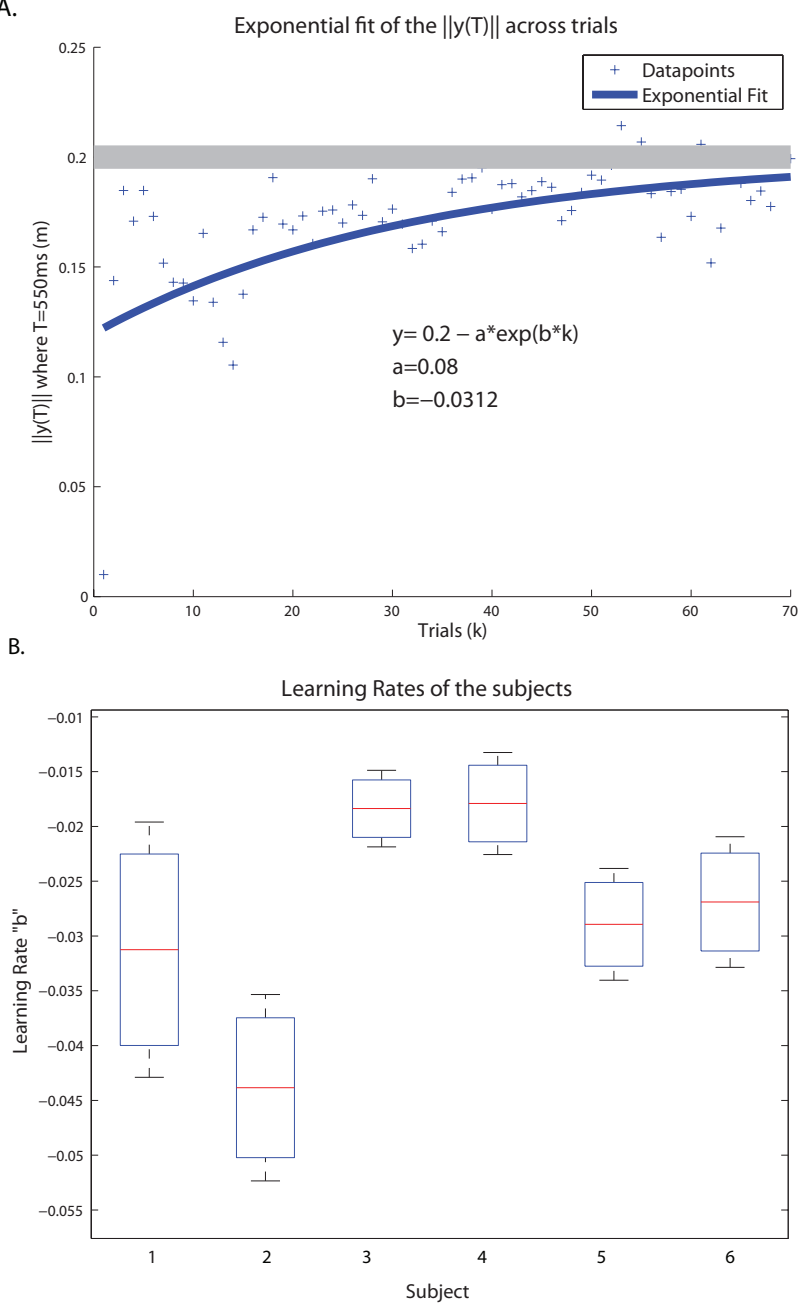

Fig. 3. A. A typical subject's position across trials at $550 \mathrm{~ms}$, along with the fitted parameters describing his/her learning rate. B. The learning rates identified (mean and standard deviation) for all subjects who conducted the experiment

where $y(k)$ is the subject's position at $550 \mathrm{~ms}$ at the $k^{\text {th }}$ trial. Positive parameters $a$ and $b$ are subsequently identified from data. In this case, the parameter $b$ represents the rate at which the subject learns the task (i.e. the convergence rate), as shown in Figure 3A. It is further observed that for all six subjects, the convergence rates are identified to be around 0:04 (Figure 3B), implying that all subjects are able to learn to improve their performance in a similar manner using the observed error between their hand position and the target. Note that due to the experimental protocol, different subject requires different number of trials in order to complete the experiment, resulting in the different learning rates observed in Figure 3 B.

In conclusion, it is observed from the experimental results that the human

1) learns to perform the reaching task using only knowledge of the endpoint, and

2) exhibits the bell-shaped velocity and accervetion profiles as seen in Figure 2 right column.

Additionally, it has been proposed that humans move in a predominantly feedforward manner with minimal contribution 
from continuous feedback within each trial [11], [12], [30].

To achieve the above observations, the problem of modelling of human motor learning using endpoint feedback is formulated as an optimization problem in the next section.

\section{General Problem Formulation}

In Section II, learning is 0 rved in experiments of requiring human subjects perfor target reaching while being provided with only endpoint visual feedback. It is shown in this section that this ability of human learning can be described by a learning controller that solves an optimization problem which balances the performance of tracking at the endpoint and that of end-effector smoothness [13].

To do this, a planar two-link manipulator model can be used to represent the human upper limb manipulating the position of the hand end-effector, by using parameters commonly adopted in the human upper limb model (see, for example, [4], [34]). This type of model is widely used in human motor learning and is consistent with the experimental setup in which the joints of the upper arm are constrained to two degrees of freedom by the robot. At each iteration, for any $t \in[0, T]$, the model of the upper limb is given as

$$
\begin{aligned}
\dot{\mathbf{x}}_{1}(t) & =\mathbf{x}_{2}(t), \\
\dot{\mathbf{x}}_{2}(t) & =\mathbf{f}\left(\mathbf{x}_{1}(t), \mathbf{x}_{2}(t)\right)+\mathbf{g}\left(\mathbf{x}_{1}(t)\right) \boldsymbol{\tau} \\
\mathbf{z}(t) & =\mathbf{h}\left(\mathbf{x}_{1}(t), \mathbf{x}_{2}(t)\right)
\end{aligned}
$$

where the states $\mathbf{x}_{1}$ and $\mathbf{x}_{2}$ are in $\mathbb{R}^{n}$ and represent the joint positions and joint velocities respectively. The input $\tau \in \mathbb{R}^{m}$ represents the driving torque; the output $\mathbf{z} \in \mathbb{R}^{p}$ is a vector containing the position and velocity states. The nonlinear mapping $\mathrm{g}: \mathbb{R}^{n} \rightarrow \mathbb{R}^{n \times m}$ represents the inverse of the inertial matrix. The nonlinear mapping $\mathrm{f}: \mathbb{R}^{n} \times \mathbb{R}^{n} \rightarrow \mathbb{R}^{n}$ is composed of the nonlinear components of the system dynamics weighted by the inverse of the inertia matrix $-\mathbf{g}\left(\mathbf{x}_{1}\right)$ and is defined as

$$
\mathbf{f}\left(\mathbf{x}_{1}, \mathbf{x}_{2}\right)=:-\mathbf{g}\left(\mathbf{x}_{1}\right)\left(\mathbf{c}\left(\mathbf{x}_{1}, \mathbf{x}_{2}\right)+\mathbf{p}\left(\mathbf{x}_{1}\right)\right),
$$

where $\mathbf{c}\left(\mathbf{x}_{1}, \mathbf{x}_{2}\right)$ and $\mathbf{p}\left(\mathbf{x}_{1}\right)$ represent the Coriolis and centrifugal effects and gravity effects respectively [34]. The nonlinear mapping $\mathbf{h}: \mathbb{R}^{n} \times \mathbb{R}^{n} \rightarrow \mathbb{R}^{p}$ is

$$
\mathbf{h}\left(\mathbf{x}_{1}, \mathbf{x}_{2}\right):=\left[\begin{array}{ll}
\mathcal{F}\left(\mathbf{x}_{1}\right) & \mathcal{J}\left(\mathbf{x}_{1}\right) \mathbf{x}_{2}
\end{array}\right],
$$

where $\mathcal{F}: \mathbb{R}^{n} \rightarrow \mathbb{R}^{p}$ represents the forward kinematics from the joint states to the end-effector and $\mathcal{J}: \mathbb{R}^{n} \rightarrow \mathbb{R}^{p}$ represents the relation between the joint and end-effector velocities. All nonlinear mappings are smooth on their domains. When performing a planar motion task, the output dimension in (2) satisfies $p=2 n=2 m$.

To model human learning, a controller needs to be designed to enable the system (2) to track a desired trajectory $\mathbf{y}_{d}(t)$ across iterations a set of finite number of points defined at various time instances $t_{j_{s}}, s=1, \ldots, M$ satisfying $0 \leq t_{j_{1}} \leq$ $\cdots \leq t_{j_{M}} \leq T$. This can be represented as a desired trajectory $\overrightarrow{\mathbf{y}}_{r}$, where

$$
\overrightarrow{\mathbf{y}}_{r}=\left[\mathbf{y}_{d}\left(t_{j_{1}}\right)^{T}, \mathbf{y}_{d}\left(t_{j_{2}}\right)^{T}, \cdots, \mathbf{y}_{d}\left(t_{j_{M}}\right)^{T}\right]^{T} \in \mathbb{R}^{p \cdot M} .
$$

Although the task considered in the experiments is only to track the endpoint, we can still formulate the human learning as a more general optimization problem where the cost function balances the tracking performance $\left(J_{t}\left(\overrightarrow{\mathbf{y}}_{r}, \boldsymbol{\tau}_{k}\right)\right)$ and other performance indices $\left(\tilde{J}\left(\boldsymbol{\tau}_{k}\right)\right.$ ) (which may be used to represent motion smoothness) through the introduction of constant weights $\alpha_{1} \geq 0$ and $\alpha_{2} \geq 0$ [25]:

$$
J\left(\overrightarrow{\mathbf{u}}_{k}\right)=\alpha_{1} J_{t}\left(\overrightarrow{\mathbf{y}}_{r}, \boldsymbol{\tau}_{k}\right)+\alpha_{2} \tilde{J}\left(\boldsymbol{\tau}_{k}\right) \quad \text { subject to (2). }
$$

Remark 1. The simulation presented in this paper is a specific application example of the problem described in this section. In this case, the simulation only attempts to model human learning of an endpoint target. Other experiments, such as those where humans are required to reach a target through via-points [15] can also be simulated using the same problem formulation.

Remark 2. The cost function (4) is versatile enough such that any important features of human motion can be included in the cost function. This paper uses motion smoothness as an illustrative example of how human motion features can be incorporated in the framework.

Through the process of solving the optimization problem (4), it is possible to construct a framework which

1) learns an optimal feedforward signal to enable it to achieve the desired task, while no online continuous feedback is required to stabilize the plant across each trial,

2) adjusts the feedforward signal at each trial to enable learning in a similar manner to the human learning using endpoint information from the last iteration,

3) possesses the capability of incorporating other possible cost that could represent factors which govern human motion, such as motion smoothness, allowing the framework to develop along with existing research in modelling human motor learning, and

4) provides flexibility that can be tailored for a particular individual by introducing new parameters $\alpha_{1}$ and $\alpha_{2}$.

To demonstrate the construction of the framework, point-topoint LC [10] is revisited in the next section.

\section{Modelling OF MOTOR LEARNING}

In the previous section, it has been shown that modelling human learning using endpoint visual feedback can be formulated as an optimization problem. This section attempts to solve the optimization problem using point-to-point LC in order to construct a framework which is representative of human learning as proposed in the literature. That is, the framework aims to model the proposition that humans learn a feedforward control action using feedback at the end of the movement of previous trial [30].

In order to construct the framework, the nonlinear dynamics given in (2) is first input-output feedback linearized [16], [17] by defining the control law $\tau$ as

$$
\begin{aligned}
\boldsymbol{\tau} & =\left(L_{g} \mathbf{h}(\mathbf{x})\right)^{-1}\left(-L_{f} \mathbf{h}(\mathbf{x})+\mathbf{u}\right) \\
& =\left(\boldsymbol{\psi}\left(\mathbf{x}_{1}\right)\right)^{-1}\left(-S\left(\mathbf{x}_{1}\right) \mathbf{f}\left(\mathbf{x}_{1}, \mathbf{x}_{2}\right)-\frac{\partial S}{\partial \mathbf{x}_{1}}\left(\mathbf{x}_{1}\right) \mathbf{x}_{2}^{2}+\mathbf{u}\right)
\end{aligned}
$$


where $S(\cdot)$ is the Jacobian of the arm forward kinematics, $\boldsymbol{\psi}(\cdot):=(S(\cdot) \mathbf{g}(\cdot))^{-1}$ and $L_{f} \mathbf{h}$ and $L_{g} \mathbf{h}$ are the Lie derivatives defined as $L_{f} \mathbf{h}(\mathbf{x}):=\frac{\partial \mathbf{f}}{\partial \mathbf{x}} \mathbf{h}(\mathbf{x})$ and $L_{g} \mathbf{h}(\mathbf{x}):=\frac{\partial \mathbf{g}}{\partial \mathbf{x}} \mathbf{h}(\mathbf{x})$ respectively [16]. This results in the following continuous-time LTI system:

$$
\begin{aligned}
\dot{\mathbf{x}}_{1} & =\mathbf{x}_{2} \\
\dot{\mathbf{x}}_{2} & =\mathbf{u} \\
\mathbf{y} & =\left[\begin{array}{ll}
\mathbf{x}_{1} & \mathbf{x}_{2}
\end{array}\right]^{T} .
\end{aligned}
$$

The system (6) is then discretized using an appropriate sampling rate $h$ to obtain an equivalent discrete-time LTI system to $(6)$ :

$$
\mathbf{y}_{k}(j+1)=A \mathbf{y}_{k}(j)+B \mathbf{u}_{k}(j), \quad j=0,1, \ldots N-1
$$

where $N$ is the total number of sampled points,

$$
A:=\left[\begin{array}{cc}
I_{n \times n} & h I_{n \times n} \\
0_{n \times n} & I_{n \times n}
\end{array}\right], \quad B:=\left[\begin{array}{c}
\frac{h^{2}}{2} I_{n \times n} \\
h I_{n \times n}
\end{array}\right] .
$$

For the experiment, the sampling rate $h$ is chosen to be $10 \mathrm{~ms}$ (100 times a second), considering that the muscle spindle firing rate is approximately $50-150 \mathrm{~Hz}$ [24] [7]. As the duration of each trial for the experiment is $550 \mathrm{~ms}$, 55 sampling points in each trial is obtained with $N=54$.

Remark 3. In general, it is required that the sampling rate $h$ should be sufficiently fast such that $N=T / h$ and the total number of points to be tracked $M$ satisfy the condition $p \cdot N-$ $\max \{d,(p-m) \cdot N\} \geq p \cdot M$, see [10] for further details. $\circ$ Considering the discrete-time LTI system (7), the sequence of inputs

$$
\overrightarrow{\mathbf{u}}_{k}=\left[\begin{array}{llll}
\mathbf{u}_{k}(0)^{T} & \mathbf{u}_{k}(1)^{T} & \ldots & \mathbf{u}_{k}(N-1)^{T}
\end{array}\right]^{T}
$$

and the sequence of outputs

$$
\overrightarrow{\mathbf{y}}_{k}=\left[\begin{array}{llll}
\mathbf{y}_{k}(0)^{T} & \mathbf{y}_{k}(1)^{T} & \ldots & \mathbf{y}_{k}(N-1)^{T}
\end{array}\right]^{T},
$$

are related by the input-output mapping $G \in \mathbb{R}^{p \cdot N \times m \cdot N}$, i.e., $\overrightarrow{\mathbf{y}}_{k}=G \overrightarrow{\mathbf{u}}_{k}$ with the resetting condition $\mathbf{y}_{k}(0)=\mathbf{0}$. This input-output mapping is also known as the transition matrix, which is defined as

$$
G=\left[\begin{array}{ccccc}
B & 0 & \cdots & \cdots & 0 \\
A B & B & 0 & \cdots & 0 \\
A^{2} B & A B & B & \cdots & 0 \\
\vdots & \vdots & \vdots & \ddots & \vdots \\
A^{N-1} B & A^{N-2} B & \cdots & \cdots & B
\end{array}\right] .
$$

The matrix (11) is used to design the cost function (4) which is subsequently solved using point-to-point LC in order to construct a framework to model human learning using the endpoint feedback from the last trial, as demonstrated in the experiments given in Section III. The two components of the cost function (i.e. the tracking and the smoothness components) are designed in the next two sections.

\section{A. Designing of the tracking component}

The cost function for the tracking component $J_{t}$ is designed in this section to ensure that the position of the hand reaches the final target at $550 \mathrm{~ms}$, thereby completing the task. For this experiment, humans are required to reach a target point $20 \mathrm{~cm}$ away from the body on the same plane with zero velocity. As such, the desired output at the target point is defined to be $\mathbf{y}_{r}=\left[\begin{array}{llll}0 & 0.2 & 0 & 0\end{array}\right]^{T}$.

Furthermore, during the experiment, humans learn to ensure that their hand position tracks the target over repeated trials using information at the end of the last trial. Therefore, only the last point of the output sequence at each trial (10) is to be considered. To incorporate this trait, as well as the dynamics of the plant described by the input-output mapping (11), the tracking component of the cost function is constructed as

$$
\min _{\overrightarrow{\mathbf{u}}} J_{t}(\overrightarrow{\mathbf{u}})=\left\|\left(\mathbf{y}_{r}-\Phi G \overrightarrow{\mathbf{u}}_{k}\right)\right\|^{2}
$$

where $\Phi \in \mathbb{R}^{p \cdot M \times p \cdot N}$ is a projection matrix defined as

$$
\Phi_{i, j}=\left\{\begin{array}{ll}
I_{p} & \text { if } j=j_{i}, \quad i=1,2, \ldots M \\
0_{p} & \text { otherwise }
\end{array},\right.
$$

which extracts a sequence of $M$ points from the output sequences. For the experiment, $M=1$ to reflect the fact that only one target is required to be reached, and the projection matrix of (13) is defined as

$$
\Phi=\left[\begin{array}{lllll}
0_{4 \times 4} & 0_{4 \times 4} & 0_{4 \times 4} & \ldots & I_{4 \times 4}
\end{array}\right]^{T} .
$$

To exhibit humans learning to track the target, a simple gradient descent method is applied to the cost function (12). This results in the feedforward sequence $\overrightarrow{\mathbf{u}}$ being adjusted at each trial $k$ according to the following law:

$$
\overrightarrow{\mathbf{u}}_{k+1}=\overrightarrow{\mathbf{u}}_{k}+\Gamma(\Phi G)^{T}\left(\overrightarrow{\mathbf{y}}_{r}-\Phi G \overrightarrow{\mathbf{u}}_{k}\right)
$$

where the update gain matrix $\Gamma$ is chosen to be

$$
\Gamma=\left[\begin{array}{cc}
400 I_{2 \times 2} & 0_{2 \times 2} \\
0_{2 \times 2} & 120 I_{2 \times 2}
\end{array}\right]
$$

to ensure convergence [10, Theorem 4].

Remark 4. The control law (14) generates an input sequence at $(k+1)^{t h}$ trial using the information of the $k^{t h}$ trial. No feedback adjustment (i.e. the information during trial $k+1$ ) is needed. Therefore, the constructed framework is comprised of a feedforward controller which learns using feedback at the end of the previous trial, and can therefore naturally be used to model human motor learning in the experiments of interest [30].

\section{B. Designing of the motion smoothness component}

Other than using a cost function $J_{t}$ to incorporate tracking performance, this section details the design of the second component of the cost function (4). This component is designed using the well-known end-effector jerk minimization [13] in order to incorporate motion smoothness into the framework. The second component of the cost function (4) is consequently designed as

$$
\tilde{J}\left(\overrightarrow{\mathbf{u}}_{k}\right)=\left\|W \Xi \overrightarrow{\mathbf{u}}_{k}\right\|^{2} \equiv\left\|\Theta \overrightarrow{\mathbf{u}}_{k}\right\|^{2},
$$


where $\Xi \in \mathbb{R}^{p \cdot N \times m \cdot N}$ is a matrix describing the first order approximation of the derivative of the acceleration of the body's end-effector in task space using the sampling rate $h$ defined in Section IV-A:

$$
\Xi=\frac{1}{h}\left[\begin{array}{ccccc}
1 & -1 & \cdots & \cdots & 0 \\
0 & 1 & -1 & \cdots & 0 \\
0 & 0 & 1 & \cdots & 0 \\
\vdots & \vdots & \vdots & \ddots & \vdots \\
0 & 0 & \cdots & \cdots & 1
\end{array}\right] .
$$

The quadratically weighted factor $W \in \mathbb{R}^{p \cdot N \times m \cdot N}$ is defined as

$$
W=\left[\begin{array}{cccc}
c_{1}^{2} I_{m \times p} & 0 & \cdots & 0 \\
0 & \left(c_{1}-c_{2}\right)^{2} I_{m \times p} & \cdots & 0 \\
\vdots & \vdots & \ddots & \vdots \\
0 & 0 & \cdots & \left(c_{1}-N c_{2}\right)^{2} I_{m \times p}
\end{array}\right]
$$

where $c_{1}$ and $c_{2}$ are two positive constants representing the bias of the non-symmetric trajectories observed from experiments. The matrix $W$ is symmetric and positive definite. This weight $W$ introduces bias into the end-effector jerk in order to account for the sharper deceleration observed in the experiment results of Figure 2 . In the simulation, $c_{1}$ and $c_{2}$ are selected heuristically as 1 and 0.1 respectively.

By incorporating a secondary objective function of weighted jerk along with the tracking objective function described in (12), the cost function (4) is defined as

$$
J=\left\|\alpha_{1}\left(\overrightarrow{\mathbf{y}}_{r}-\Phi \overrightarrow{\mathbf{y}}_{k}\right)\right\|^{2}+\|\left.\alpha_{2} \Theta \overrightarrow{\vec{\Omega}}\right|^{2},
$$

where positive constants $\alpha_{1}$ and $\alpha_{2}$ are weights on tracking and jerk components respectively. Using gradient decent, the following update law is derived for the framework:

$$
\begin{aligned}
\overrightarrow{\mathbf{u}}_{k+1}= & \overrightarrow{\mathbf{u}}_{k}+\Upsilon \alpha_{1}^{2}(\Phi G)^{T}\left(\overrightarrow{\mathbf{y}}_{r}-\Phi \overrightarrow{\mathbf{y}}_{k}\right) \\
& -\Upsilon \alpha_{2}^{2} \Theta^{T} \Theta \overrightarrow{\mathbf{u}}_{k},
\end{aligned}
$$

where $\Upsilon$ is an updating gain matrix with appropriate dimension. By applying [10, Theorem 4], the convergence of the updating law (18) can be guaranteed if $\Upsilon$ satisfies

$$
\begin{aligned}
& \underline{\sigma}(\Upsilon)>0 \\
& \bar{\sigma}(\Upsilon)<\min \left\{\frac{2}{\bar{\sigma}\left(\left(\alpha_{2} R\right)\left(\alpha_{2} R\right)^{T}\right)}, \frac{2}{\bar{\sigma}\left(\alpha_{1} \Phi G\left(\alpha_{1} \Phi G\right)^{T}\right)}\right\},
\end{aligned}
$$

where $\underline{\sigma}(A)$ and $\bar{\sigma}(A)$ are respectively the smallest and the largest singular values of a possibly non-square matrix $A$ [35], and the matrix $R$ is selected as $R=\left[\begin{array}{c}\Phi G \\ -\Theta^{T} \Theta\end{array}\right]$.

In the simulation model, the update matrix is chosen as

$$
\Upsilon=\left[\begin{array}{cc}
400 I_{2 \times 2} & 0_{2 \times 2} \\
0_{2 \times 2} & 120 I_{2 \times 2}
\end{array}\right]
$$

to ensure the convergence of the tracking error.

The weights $\alpha_{1}$ and $\alpha_{2}$ produce a balance between the tracking performance and the smoothness of the acceleration signals. Different choices of these two parameters leads to different performance. Considering that different people have different weights, the obtained experimental data is used to find an appropriate ratio between $\alpha_{1}$ and $\alpha_{2}$. The ratio can be derived by considering (18) at steady state:

$$
\Upsilon\left(\alpha_{1}^{2}(\Phi G)^{T}\left(\overrightarrow{\mathbf{y}}_{r}-\Phi \overrightarrow{\mathbf{y}}_{s s}\right)-\alpha_{2}^{2}\left(\Theta^{T} \Theta \overrightarrow{\mathbf{u}}_{s s}\right)=0,\right.
$$

where $\overrightarrow{\mathbf{u}}_{s s}$ and $\overrightarrow{\mathbf{y}}_{s s}$ are the steady state input and output which are observed after the human has learned the movement in the experiment respectively (i.e. $\overrightarrow{\mathbf{u}}_{k+1}=\overrightarrow{\mathbf{u}}_{k}=\overrightarrow{\mathbf{u}}_{s s}$ and $\overrightarrow{\mathbf{y}}_{k}=\overrightarrow{\mathbf{y}}_{k+1}=\overrightarrow{\mathbf{y}}_{s s}$ ) for a sufficiently large $k$. Considering that the weights are scalars, the ratio $\rho:=\frac{\alpha_{2}^{2}}{\alpha_{1}^{2}}$ can subsequently be determined as

$$
\rho=\frac{\left\|(\Phi G)^{T}\left(\mathbf{y}_{r}-\Phi \mathbf{y}_{s s}\right)\right\|}{\left\|\Theta^{T} \Theta \mathbf{u}_{s s}\right\|} .
$$

Using the experiment data, the ratio $\rho$ is computed as $\rho=5.1740 \times 10^{-4}$. This indicates that the primary objective (tracking) is dominant which is consistent with the experimental setting. In order to show the influence of minimal jerk, $\alpha_{1}$ is selected as 100 in the simulation. This leads to the value of $\alpha_{2}=0.517$, which is similar to the values determined heuristically.

Remark 5. Similar to [10, Section III-B], it is possible to reformulate the reaching task as an optimization problem with a hard constraint on tracking performance. That is, subjects must be able to reach the endpoint at exactly $550 \mathrm{~ms}$ at every trial. However, such problem formulation is not consistent with what was observed during experiments as the tracking error of the endpoint at $550 \mathrm{~ms}$ is adjusted at every trial by the human (see Figure 2). In addition, such formulation makes it difficult to determine the importance of objectives such as jerk minimisation compared to the objective of endpoint tracking when the subject performs the reaching task. In contrast, the problem formulation presented makes it possible to find, through comparison with experiment data, that humans weigh the tracking objective more highly than the minimization of jerk. That is, although smoothness is an important characteristic of human motion, humans are willing to trade it off in order to track a given task.

Remark 6. The current framework assumes that humans possess perfect knowledge of the body. However, the framework is also applicable for the case where there are imperfections in humans' knowledge of the body and of the environment. This is because the point-to-point LC implemented in this framework is robust to modelling uncertainties and disturbances [10]. The framework can therefore ensure learning of the target reaching task even if the model dynamics (2) are different from the actual dynamics.

Remark 7. In previous work, inverse optimal control has been applied to steady-state experiment data in order to identify the weights of a cost function describing the human's motion [2], [3], [22]. This approach is designed to model human motions without repetitions and does not have the focus on the "learning" aspect of human motor control. On the other hand, the proposed point-to-point learning control is able to adjust its input through repetitions by solving a standard optimization problem with a high-dimensional input using the gradient descent method. This kind of problem formulation enables 
the controller to model human's ability to learn through repetitions, and is therefore more applicable to model human learning using endpoint feedback. The model is therefore shown to be able to not only identify the necessary parameters to model human motions at the end of learning (steady state over iterations), but also the motions during learning (transient across iterations) through the appropriate choice of the update gain of the gradient descent (i.e. $\Upsilon$ in (18)).

\section{Simulation Results}

In the previous section, it is demonstrated that by solving an optimization problem of the form (4), a framework using point-to-point LC can be constructed to simulate the task of learning to reach a target using only endpoint visual feedback. In this section, the experiment results are compared to the simulation results obtained using the control laws described in (14) and (18) of Sections IV-A and IV-B, representing the cases of with and without the consideration of human movement smoothness respectively.

First of all, a general qualitative appreciation of the simulation and experiment results is made by comparing the simulation and experimental trajectories over the first 10 trials (before learning) and over the last 10 trials (after learning). A quantitative comparison is then made by observing how well the model predicts the progression in tracking accuracy as the number of trials increases, and by evaluating how well the simulated trajectories match the subject's experimental trajectories during each trial.

\section{A. Qualitative comparison}

The subject's movements during the first 10 trials and during the last 10 trials of the experiment are presented in Figure 4, Column 3. Simulations obtained from the models described in Section III are shown in Figure 4 Columns 1 and 2. It is observed that the position profiles produced by the simulation are able to track the final target independent of the inclusion of motion smoothness. During early trials, the trajectories show that the path which the hand follows experiences difficulties in reaching the target but over the last 10 trials, the hand is observed to be able to reach the target (Figure 4, Columns 1 and 2). This is unsurprising considering that the main objective for both models is to minimize the difference between the hand position at the end of each movement and the target. This learning is also observed in the experiment results (Figure 4, Column 3). For both simulations, the velocity profile is bellshaped, which is similar to that of the experiment (Figure 4, Column 3). Therefore, if only position and velocity profiles are considered, both models are able to simulate how humans track the final target point using only endpoint information.

On the other hand, significant differences between the acceleration profiles are observed for the two simulations. When considering only tracking and not motion smoothness, it is observed that the acceleration profile obtained through the simulation failed to match that of the experimental data (Figure 4, Column 1). The simulation predicts instantaneous increase of the acceleration of over $3 \mathrm{~m} / \mathrm{s}^{2}$, which is not supported by the observation of gradual acceleration increase shown by the
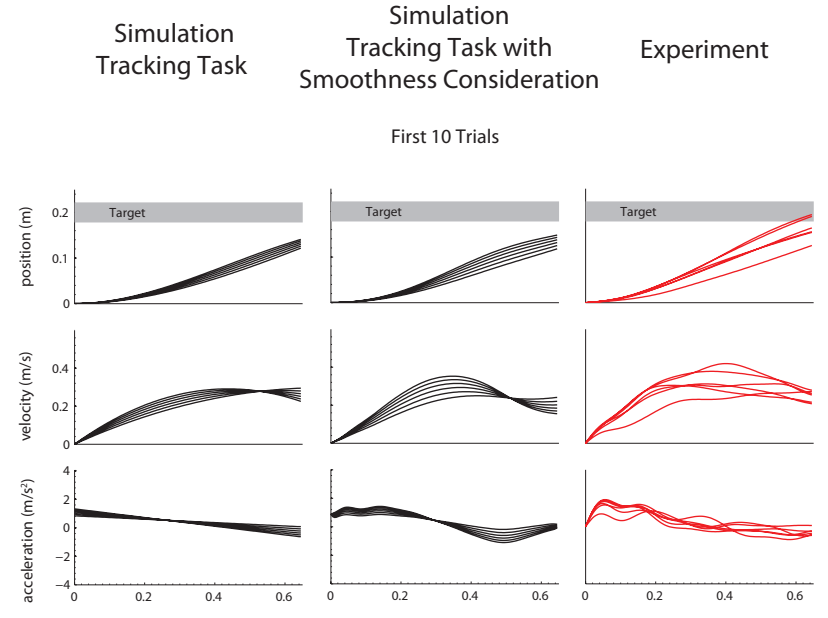

Last 10 Trials
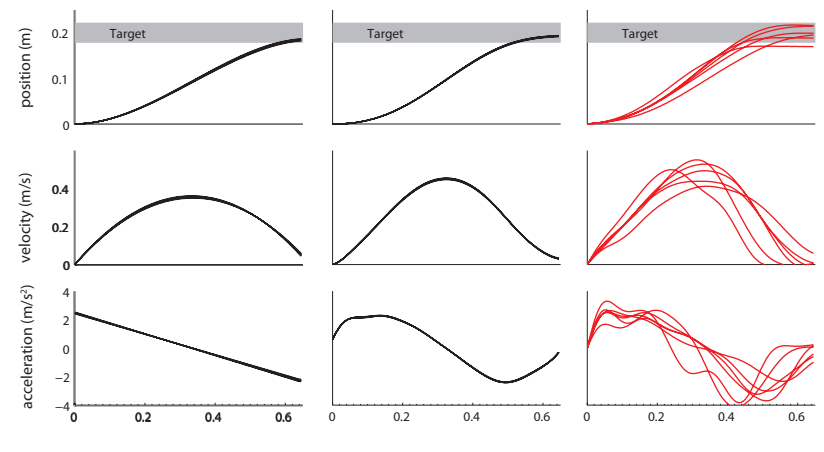

time (s)

Fig. 4. Comparison between simulation results from each of the two stages outlined in Section IV-A (column 1) and Section IV-B (column 2) and experiment results (column 3) for the vertical $y$ direction. This shows that by incorporating motion smoothness, the simulation is able to better capture the experimental observations (column 2 vs column 3 ) when compared to the case where only tracking is considered (column 1)

human subject. When motion smoothness is considered, the ratio $\rho$ is estimated from experiments as $\rho=5.1740 \times 10^{-3}$. This ratio indicates that jerk is not the dominant objective of the cost function compared to tracking the target. Nevertheless, by choosing the cost function weights as $\alpha_{1}=100$ and $\alpha_{2}=0.517$ to reflect the ratio $\rho$, the simulation model is able to generate trajectories which converges to the target with a bell-shaped velocity profile and a smooth acceleration profile (Figure 4, Column 2). More specifically, the acceleration profile exhibits a gradual increase to the maximum value before de-accelerating gradually to the maximum deceleration, which more closely matches experimental rewlts.

Remark 8. The simulation results converges to a smooth distinct trajectory while variations continue to be observed in the experimental results in Figure 4, even over the last 10 trials. The fluctuations are due to human variability, which can be simulated by introducing white noise to the plant model [34]. This is not done in this paper because the purpose of this work is to demonstrate the framework's ability to model human learning using endpoint information alone, and the ability to incorporate some human motion characteristics such as smoothness into the cost function. 


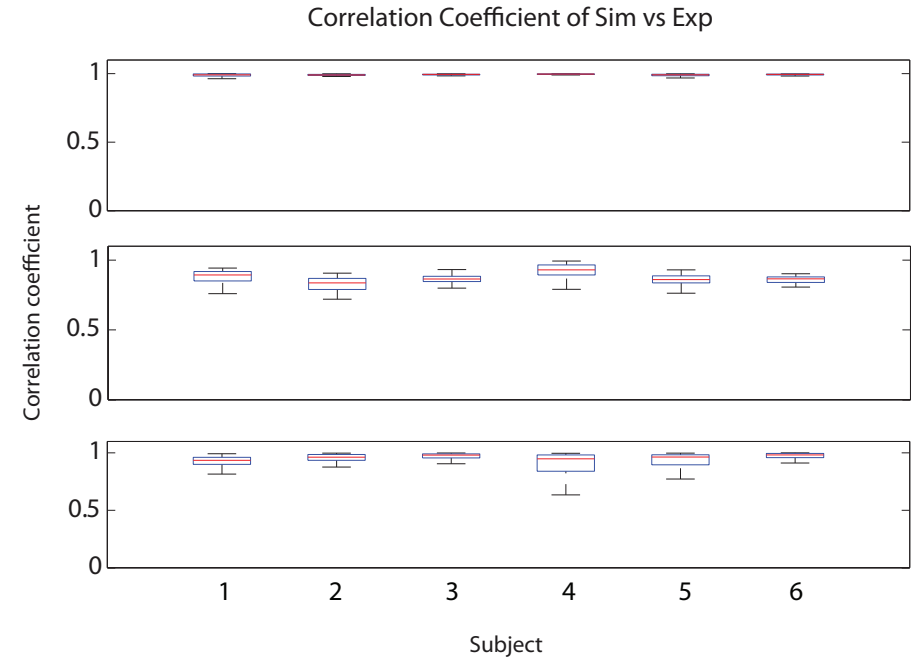

Fig. 5. Comparison of accuracy (left column) during the experiment (.) and that simulated by the model $(+)$ (far left column, Figure 5A and B: Accuracy in Reaching the Target). It is observed that independent of the inclusion of motion smoothness, the simulation is able to display learning convergence towards the target at the endpoint in a similar manner to that of the subject. However, it is evident that the incorporation of motion smoothness (Figure 5 D) provides a correlation coefficient close to 1 for the majority of the trials compared to the case when the simulation only considers tracking (Figure 5 C)

\section{B. Quantitative comparison}

In this section, simulation results constructed from the models described in Section III are compared quantitatively to the experiment results described in Figure 5. For the reaching task, the subject's hand position at the end of each trial during the experiment is compared to that of the simulation (Figure 5, A and B). From these graphs, it is observed that the simulation results show convergence of the hand's endpoint over trials independent of the inclusion of motion smoothness. This agrees with the fact that tracking the target is the main objective considered by the two models and demonstrates the framework's effectiveness in exhibiting learning using feedback at the end of each trial.

To observe how well the simulated trajectories compare to those of the experiments at each trial, the associated correlation coefficient for each trial is plotted in Figure 5 (C and D). Comparing the two figures, it is evident that without consideration of motion smoothness, the simulation exhibits lower performance in matching experimental results. This is especially significant when observing the correlations between the velocity and the acceleration profiles.

To observe how well the simulation applies to the entire population, the framework is applied to all six subjects' data and the correlations between the simulated and experiment results are obtained. The box plots of the correlations (Figure 6) shows that the model is able to simulate the experiment results well for all six subjects, with the correlation coefficients for position and acceleration being above 0.7 , while the velocity is above 0.5 . The coefficients are similar to those of other models reported in the literature [4], [34].

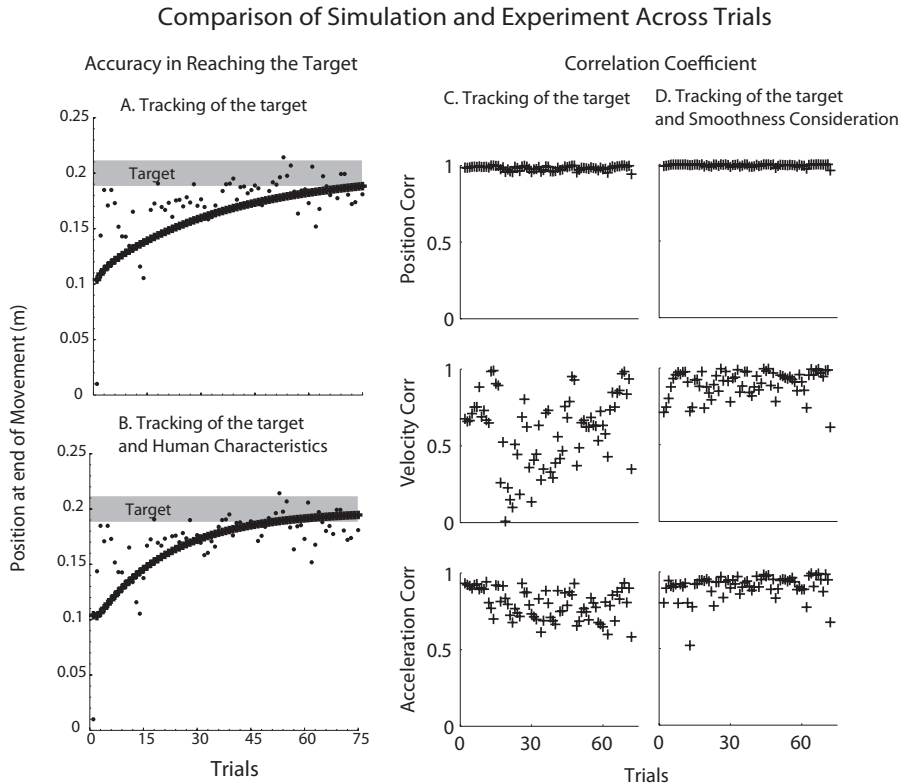

Fig. 6. Comparison of correlation coefficient for all six subjects. It is observed that the simulation is able to consistently perform well in predicting the experiment data for all six subjects for both position, velocity and acceleration

\section{Discussion AND CONCLUSION}

Humans can learn point-to-point reaching using only visual feedback of their cursor position at the end of each trial [9], [26]. Learning in such task is unaffected whether continuous visual feedback is provided or only movement endpoint visual feedback is available. Computational models to date, however, always consider human learning along the entire trajectory of the motion, assuming the availability of feedback across the entire movement [4], [8], [21], [34]. Consequently they are unable to model the experiment observations and concepts for the case where only the endpoint is provided at the end of the movement.

This current paper shows how human learning using endpoint visual feedback can be posed as an optimization problem where the cost function weighs the tracking of the endpoint and motion smoothness. It is further demonstrated through solving the proposed optimization problem using point-topoint LC that it is possible to construct a framework which is representative of the methods in which humans learn the experimental task. More specifically, the framework is able to

1) Model the human ability to learn a feedforward controller along the iteration domain.

2) Model human ability to learn using only information provided at the end of each trial, as opposed to continuous feedback information.

3) Incorporate additional factors which govern human motion such as smoothness of motion.

It is observed in Section $\mathrm{V}$ that the constructed framework is able to exhibit good consistency between the simulations and the experiments. Noting that the computational model has been previously shown to successfully model human motor learning using feedback from the entire trajectory [35], it is therefore demonstrated to capture the observation that for a 
target reaching task, human learning ability is unaffected in learning using the feedback throughout the entire trajectory or using only the feedback at the end of the movement [9].

Furthermore, the ability to include additional factors and the simplicity of the current framework provides it with a significant versatility in simulating human motion. The same framework can also be applied to human learning in other experiments, such as movement through via-points [15], or movement in three-dimensions through the addition of an extra dimension to the dynamic system. The main challenges which the authors can foresee when modelling novel experiments is the design of the experiment itself. For example, to extend the framework to model three dimensional movement, suitable motion capture mechanisms are required, while the experimenter needs to design suitable constraints to restrict the subject's motion such that it can be accurately represented by a simplified model of the arm.

Further experiments can be modelled by applying small changes to the structure of the optimization problem of Section III. For example, by simply changing the value of the target location $\left(\vec{y}_{r}\right)$ of (3), the framework can be used to model the experiment of humans learning multiple targets in multiple directions as outlined in the literature [26]. In the same way, it can be used to simulate human reaching motion to different parts of the workspace. Any differences in the human motion dynamics as a result of the different workspace can be easily reflected by the different values of identified weights $\alpha_{1}$ and $\alpha_{2}$. Future work will attempt to formulate other experiments in the literature as optimization problems, such as those involving different environmental forces and visions, and solving them to simulate human learning as observed in the literature. By doing this, it is hoped that the constructed frameworks can aid motor control scientists to further develop the theory of human motor learning.

\section{ACKNOWLEDGMENT}

The authors would like to thank...

\section{REFERENCES}

[1] M. Berniker and K. Kording, "Estimating the sources of motor errors for adaptation and generalization," Nature Neuroscience, vol. 11, no. 12, pp. 1454-1461, Dec. 2008.

[2] B. Berret, E. Chiovetto, F. Nori, and T. Pozzo, "Evidence for composite cost functions in arm movement planning: An inverse optimal control approach," PLoS Computational Biology, vol. 7, no. 10, p. e1002183, Oct 2011

[3] B. Berret, C. Darlot, F. Jean, T. Pozzo, C. Papaxanthis, and J. P. Gauthier, "The inactivation principle: mathematical solutions minimizing the absolute work and biological implications for the planning of arm movements," PLoS Comput Biol, vol. 4, no. 10, p. e1000194, 2008.

[4] E. Burdet, K. P. Tee, I. Mareels, T. E. Milner, C. M. Chew, D. W. Franklin, R. Osu, and M. Kawato, "Stability and motor adaptation in human arm movements." Biological Cybernetics, vol. 94, no. 1, pp. 20 32, 2006.

[5] F. Burdet and Milner, Human robotics: neuromechanics and motor control. Upper Saddle River, N.J: MIT Press (in press), 2013.

[6] J. D. Connolly and M. A. Goodale, "The role of visual feedback of hand position in the control of manual prehension." Experimental Brain Research, vol. 125, no. 3, pp. 281-6, 1999.

[7] P. E. Crago, J. C. Houk, and W. Z. Rymer, "Sampling of total and muscle force and by tendon and organs," Journal of Neurophysiology, vol. 47, pp. 1069-83, 1982.
[8] D. W. Franklin, E. Burdet, K. P. Tee, R. Osu, C.-M. Chew, T. E. Milner, and M. Kawato, "CNS learns stable, accurate, and efficient movements using a simple algorithm." The Journal of Neuroscience, vol. 28, no. 44, pp. 11 165-73, 2008.

[9] D. W. Franklin, U. So, E. Burdet, and M. Kawato, "Visual feedback is not necessary for the learning of novel dynamics." PloS one, vol. 2, no. 12, p. e1336, 2007.

[10] C. T. Freeman and Y. Tan, "Iterative Learning Control With Mixed Constraints for Point-to-Point Tracking," IEEE Transactions on Control Systems Technology, vol. 21, no. 3, pp. 604-616, 2012.

[11] M. Heath, D. Westwood, and G. Binsted, "The control of memoryguided reaching movements in peripersonal space." Motor control, 2004.

[12] M. R. Hinder, S. Riek, J. R. Tresilian, A. de Rugy, and R. G. Carson, "Real-time error detection but not error correction drives automatic visuomotor adaptation." Experimental Brain Research, vol. 201, no. 2, pp. 191-207, Mar. 2010.

[13] N. Hogan and T. Flash, "The coordination of arm movements: an experimentally confirmed mathematical model," The Journal of Neuroscience, vol. 5, no. 7, pp. 1688-1703, 1985.

[14] H. A. Ingram, P. van Donkelaar, J. Cole, J. L. Vercher, G. M. Gauthier, and R. C. Miall, "The role of proprioception and attention in a visuomotor adaptation task." Experimental Brain Research, vol. 132, no. 1, pp. 114-26, 2000

[15] J. Izawa, T. Rane, O. Donchin, and R. Shadmehr, "Motor adaptation as a process of reoptimization," Journal of Neuroscience, vol. 28, no. 11, pp. 2883-2891, Mar. 2008.

[16] H. K. Khalil, Nonlinear systems, 3rd ed. Upper Saddle River, N.J: Prentice Hall, 2002.

[17] O. Khatib, "A Unified Approach for Motion and Force Control of Robot Manipulators: The Operational Space Formulation," IEEE Journal of Robotics and Automation, vol. RA-3, no. 1, pp. 43-53, 1987.

[18] J. Klein, N. Roach, and E. Burdet, "3dom: a 3 degree of freedom manipulandum to investigate redundant motor control," Haptics, IEEE Transactions on, vol. 7, no. 2, pp. 229-239, 2014.

[19] K. P. Koding and D. M. Wolpert, "Bayesian decision theory in sensorimotor control," Trends in Cognitive Sciences, vol. 10, no. 7, pp. 319-326, Jul. 2006

[20] J. W. Krakauer, "Motor learning: its relevance to stroke recovery and neurorehabilitation." Current Opinion in Neurology, vol. 19, no. 1, pp. 84-90, 2006.

[21] W. Li and E. Todorov, "Iterative linear quadratic regulator design for nonlinear biological movement systems." in 1st International Conference on Informatics in Control, Automation and Robotics, 2004, pp. 222-229.

[22] W. Li, E. Todorov, and D. Liu, "Inverse optimality design for biological movement systems," in World Congress, vol. 18, no. Part 1, 2011.

[23] A. Mason and B. Bernardin, "The role of visual feedback when grasping and transferring objects in a virtual environment," in Proceedings of the 5th International Conference on Enactive Interfaces, 2008, pp. 111-116.

[24] M. P. Mileusnic, I. E. Brown, N. Lan, and G. E. Loeb, "Mathematical models of proprioceptors. i. control and transduction in the muscle spindle," Journal of Neurophysiology, vol. 96, no. 4, pp. 1772-1788, 2006.

[25] S. Mishra, U. Topcu, and M. Tomizuka, "Optimization-based constrained iterative learning control," IEEE Transactions on Control Systems Technology, vol. 19, no. 6, pp. 1613-1621, 2011.

[26] R. A. Scheidt, M. A. Conditt, E. L. Secco, F. A. Mussa-ivaldi, and A. Robert, "Interaction of visual and proprioceptive feedback during adaptation of human reaching movements," Journal of Neurophysiology, vol. 93 , no. 6 , pp. 3200-3213, Jun 2005.

[27] S. J. Sober and P. N. Sabes, "Flexible strategies for sensory integration during motor planning." Nature neuroscience, vol. 8, no. 4, pp. 490-7, Apr. 2005.

[28] K. Tee, D. Franklin, M. Kawato, T. Milner, and E. Burdet, "Concurrent adaptation of force and impedance in the redundant muscle system," Biological Cybernetics, vol. 102, no. 1, pp. 31-44, 2010.

[29] K. A. Thoroughman and R. Shadmehr, "Learning of action through adaptive combination of motor primitives," Letters to Nature, vol. 407, pp. 742-747, Oct. 2000.

[30] Y.-W. Tseng, J. Diedrichsen, J. W. Krakauer, R. Shadmehr, and A. J. Bastian, "Sensory prediction errors drive cerebellum-dependent adaptation of reaching," Journal of Neurophysiology, vol. 98, no. 1, pp. 54-62, May 2007. [Online]. Available:

[31] R. J. van Beers, "Motor learning is optimally tuned to the properties of motor noise," Neuron, vol. 63, no. 3, pp. 406-417, Aug. 2009.

[32] D. M. Wolpert, J. Diedrichsen, and J. R. Flanagan, "Principles of sensorimotor learning." Nature reviews. Neuroscience, vol. 12, no. 12, pp. 739-51, Dec. 2011. 
[33] S.-H. Zhou, D. Oetomo, Y. Tan, I. Mareels, and E. Burdet, "Effect of sensory experience on motor learning strategy," Journal of Neurophysiology, Nov 2014.

[34] S.-H. Zhou, D. Oetomo, Y. Tan, E. Burdet, and I. Mareels, "Modeling individual human motor behavior through model reference iterative learning control." IEEE Transactions on Biomedical Engineering, vol. 59, no. 7, pp. 1892-901, 2012.

[35] S.-H. Zhou, Y. Tan, D. Oetomo, C. Freeman, E. Burdet, and I. Mareels, "Point-to-point learning in human motor systems", in 2013 American Control Conference (ACC) Washington, DC, USA, June 17-19, 2013, 2013.

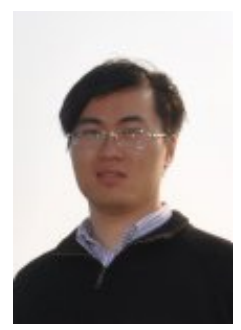

Shou-Han Zhou Shou-Han Zhou received his BEng degree, M.S degree and $\mathrm{PhD}$ degree all from the Department of Mechanical Engineering, the University of Melbourne. He is currently a post-doctorate at Imperial College London, UK. His main interests are computation neuroscience, adaptive control systems and robotics.

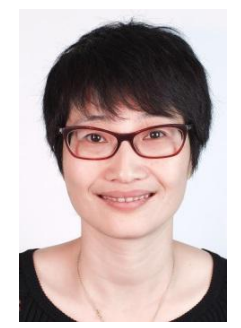

Ying Tan Dr Ying Tan is an Associate Professor and Reader in the Department of Electrical and Electronic Engineering (DEEE) at The University of Melbourne, Australia. She is also Assistant Dean, international exchange for Melbourne School of Engineering (MSE). Dr Tan received her Bachelor's degree from Tianjin University, China, in 1995, and her $\mathrm{PhD}$ from the National University of Singapore in 2002. She joined McMaster University in 2002 as a postdoctoral fellow in the Department of Chemical Engineering. Since 2004, she has been with the University of Melbourne. She was awarded an Australian Postdoctoral Fellow (2006-2008) and a Future Fellow (2009-2013) by the Australian Research Council. Her research interests are in intelligent systems, nonlinear control systems, real time optimisation, sampled-data distributed parameter systems and formation control.

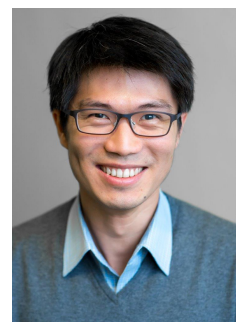

Denny Oetomo Denny Oetomo graduated BEng (Hons 1) from the Australian National University in 1997 and $\mathrm{PhD}$ from the National University of Singapore in 2004. He joined the University of Melbourne in Jan 2008, where he is currently an Associate Professor. His main research interests are in the manipulation strategies of robotics systems, with a focus on the biomedical and clinical applications.

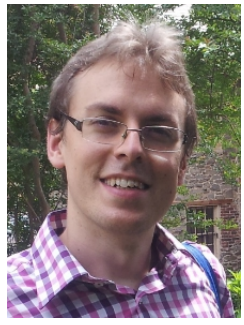

Chris Freeman Chris Freeman received his BEng degree in Electromechanical Engineering in 2000, the $\mathrm{PhD}$ degree in applied control in 2004, both from the University of Southampton, UK, and BSc degree in Mathematics from the Open University, UK, in 2006. He is currently Associate Professor in Electronics and Computer Science at the University of Southampton, UK. He has published 230 journal and conference papers primarily on the development, application and assessment of intelligent controllers within both the biomedical engineering domain and

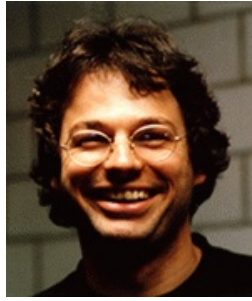

Etienne Burdet Etienne Burdet received the M.S degree in mathematics, the M.S. degree in physics, and the Ph.D. degree in robotics from the Swiss Federal Institute of Technology, Zurich, Switzerland, in 1990, 1991, and 1996, respectively. He is currently Chair in Human Robotics at Imperial College London, UK, and a visiting Professor at Nanyang Technological University in Singapore. He is doing research at the interface of robotics and bioengineering and his main interest is in humanmachine interaction. He has contributions in fields from human motor control to virtual-reality-based training systems, assistive devices, and robotics for life sciences.

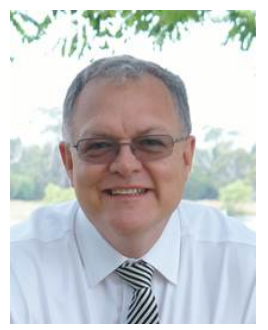

Iven Mareels Iven Mareels is Dean of the School of Engineering, the University of Melbourne. He is a Redmond Barry Distinguished Professor holding the Chair of Electrical and Electronic Engineering. He obtained the Masters of Electromechanical Engineering from Gent University Belgium in 1982 and the $\mathrm{PhD}$ in Systems Engineering from the Australian National University in 1987. He is a Fellow of The Academy of Technological Sciences and Engineering (ATSE) Australia; The Institute of Electrical and Electronics Engineers (USA), the International Federation of Automatic Control and Engineers Australia. He is a Foreign Member of the Royal Flemish Academy of Belgium for Science and the Arts. Iven has published widely in the area of adaptive and nonlinear control systems, with applications to large scale systems such as water distribution systems and the electricity grid as well as biomedical engineering applications in rehab engineering and epilepsy. 